\title{
TMAO: protecting proteins from feeling the heat
}

Authors: Mayank Boob ${ }^{1}$, Shahar Sukenik ${ }^{2,7}$, Martin Gruebele ${ }^{*}, 1-3$ and Taras Pogorelov ${ }^{*}, 1,2,4,5,6$

Affiliations:

${ }^{1}$ Center for Biophysics and Quantitative Biology, University of Illinois at Urbana-Champaign, Urbana, IL 61801, United States.

${ }^{2}$ Department of Chemistry, University of Illinois at Urbana-Champaign, Urbana, IL 61801, United States.

${ }^{3}$ Department of Physics, University of Illinois at Urbana-Champaign, Urbana, IL 61801, United States.

${ }^{4}$ School of Chemical Sciences, University of Illinois at Urbana-Champaign, Urbana, IL 61801, United States.

${ }^{5}$ Beckman Institute for Advanced Science and Technology, University of Illinois at UrbanaChampaign, Urbana, IL 61801, United States.

${ }^{6}$ National Center for Supercomputing Applications, University of Illinois at Urbana-Champaign, Urbana, IL 61801

${ }^{7}$ Present address: Department of Chemistry and Chemical Biology, UC Merced, Merced, CA 95344, United States.

*To whom correspondence may be addressed: Email: mgruebel@illinois.edu or pogorelo@illinois.edu, 


\begin{abstract}
Osmolytes are ubiquitous in the cell and play an important role in controlling protein stability under stress. The natural osmolyte trimethylamine $\mathrm{N}$-oxide (TMAO) is used by marine animals to counteract the effect of pressure denaturation at large depths. The molecular mechanism of TMAO stabilization against pressure and urea denaturation has been extensively studied, but the effect of TMAO against high temperature has not been addressed. To delineate the effect of TMAO on folded and unfolded ensembles at different temperatures, we study a mutant of the well-characterized, fastfolding model protein B (PRB). We have carried out extensive, $>190 \mu \mathrm{s}$ in total, all-atom simulations of thermal folding/unfolding of PRB at multiple temperatures and concentrations of TMAO. The simulations captured folding and unfolding events and show an increased stability of PRB in presence of TMAO. At higher TMAO concentration, intermediate ensembles are gradually more favored over the unfolded state. Quantifying TMAO-water interactions revealed that at a low concentration threshold, TMAO forms a shell near but not at the protein surface, disrupting the water network and increasing hydration of the protein to help stabilize it. Intriguingly, we found that there are intermittent interactions between TMAO and certain protein side chains with preferred TMAO orientations. Although previous studies have proposed such interactions, the long time scales we study here help to highlight the protein's sensitivity to local environment, particularly hydration, and raise questions about how even transient interactions could couple protein stability to TMAO effects.
\end{abstract}

TOC graphic:

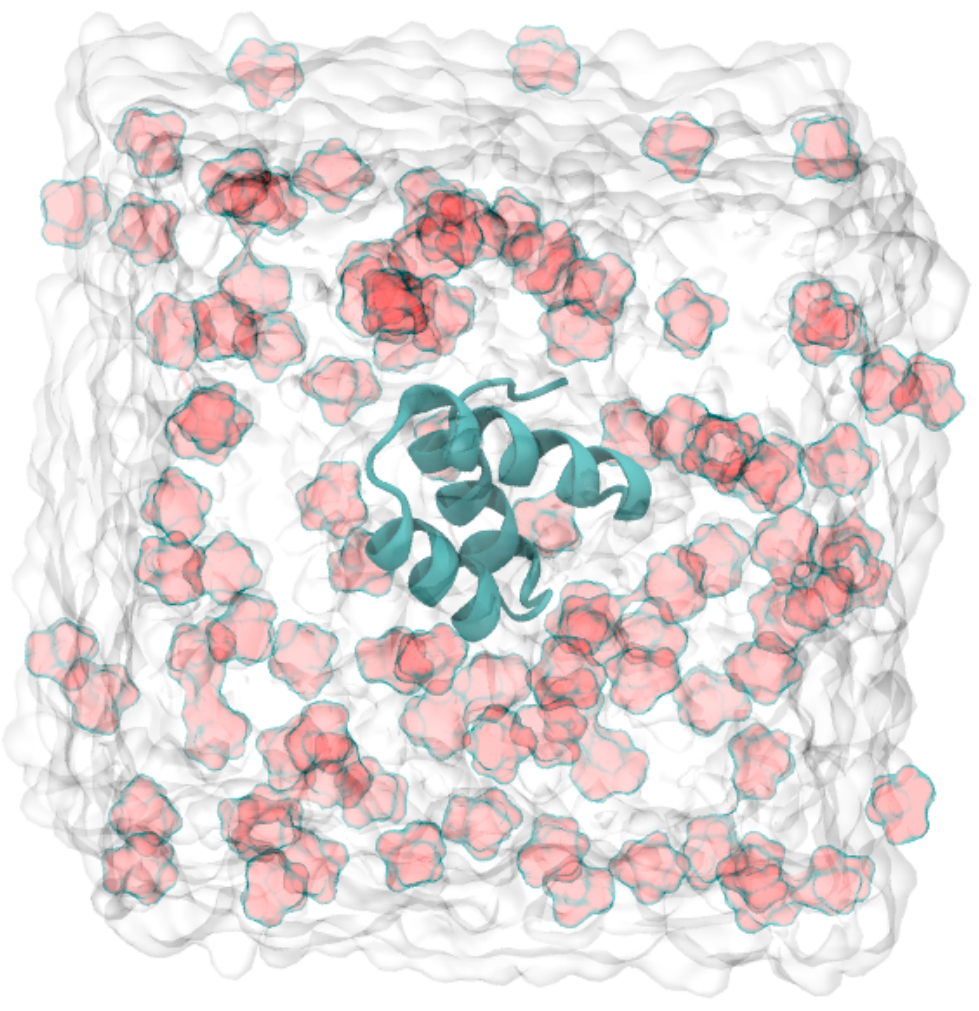




\section{Introduction}

Osmolytes are small organic molecules that regulate the overall cellular composition and maintain fluid balance in the cell. Other than their role in maintaining structural integrity they have been shown to be important for protein folding and stability. ${ }^{1}$ Osmolytes can be broadly divided into chaotropes ('structure breakers') and kosmotropes ('structure makers'). ${ }^{2}$ The mechanism of stabilization or destabilization of protein structure by these osmolytes depends on their interaction with the hydrogen bond network of water and also their direct interaction with the protein. ${ }^{3}$ Destabilizing osmolytes generally strongly interact with the protein backbone while stabilizing osmolytes are excluded from the protein surface. ${ }^{4}$

Trimethylamine $\mathrm{N}$-Oxide (TMAO) is a non-ionic kosmotrope known to stabilize proteins against chemical and pressure denaturation. TMAO has been shown to stabilize against denaturation by urea in a 2:1 ratio indicating that $1 \mathrm{M}$ TMAO is enough to protect the protein against denaturation by $2 \mathrm{M}$ Urea in solution. ${ }^{5,6}$ On the other hand, the amount of TMAO increases almost linearly with depth in the bodies of fishes indicating its role in stabilizing proteins against effects of hydrostatic pressure. ${ }^{7}$ The mechanism by which TMAO protects proteins against urea is distinct from the way it protects proteins against pressure., , $^{8}$ rea interacts directly with the protein backbone and this preferential interaction is what causes the unfolding of the protein. ${ }^{10-13}$ In the presence of TMAO, urea preferentially interacts with the TMAO instead of the protein backbone. ${ }^{14-16}$ TMAO is excluded from the protein surface, and studies of crowding due to TMAO show that the stabilization may have both significant enthalpic or entropic contributions. ${ }^{17}$ Since TMAO is generally repelled from the protein surface, despite indications of some residue-specific interactions, the effect on protein stability is assumed to be mediated by its effect on the water structure around protein. ${ }^{8,11,18}$

TMAO is unique due to the partial charge separation on the nitrogen and oxygen and its "nonhydrophobic" methyl groups. The oxygen has been shown to form three hydrogen bonds with its surroundings and the methyl groups have interactions with the oxygen of water. ${ }^{19-21}$ While there is some consensus on the effect of stabilization by TMAO in cases of chemical and pressure denaturation, little is known about its effect on temperature denaturation. In particular, the interaction of TMAO and water at higher temperature has not been studied in detail. The unfolding of proteins due to temperature is distinct from chemical and pressure denaturation. ${ }^{22}$ Proteins undergo both cold and heat denaturation, ${ }^{23}$ due to loss of hydrophobic interactions at low temperature,${ }^{24}$ and increased configurational entropy of the chain at high temperature. ${ }^{25}$ While the effect of temperature on protein structure is well studied, here we are using all-atom molecular dynamics simulations to understand the effects of TMAO on temperature denaturation of proteins.

The protein that we choose for our study is Protein $B$ (PRB), a small albumin-binding domain consisting of 47 amino acids. ${ }^{26}$ The variant consists of three alpha helices and has been mutated to fold on the microsecond time scale. ${ }^{27}$ This makes it ideal for probing its interaction with TMAO on a longer timescale than before. ${ }^{13,28,29}$ Previous studies have shown that the folding timescale of PRB is around $4 \mu \mathrm{s}$ in both experiments and simulations. ${ }^{27,30}$ Performing all-atom microsecond simulations at different temperatures and TMAO concentrations (12 conditions modeled for $\sim 16 \mu \mathrm{s}$, each, for the total time of $\sim 190 \mu \mathrm{s}$ ), enables us to gain insights into how TMAO affects water structure and interacts with the protein at different temperatures and different concentrations of TMAO. 


\section{Methods}

Simulation setup. All-atom simulations of PRB were carried out using the GROMACS 5.1.2 $2^{31}$ simulation package. Four different temperatures $(340 \mathrm{~K}, 350 \mathrm{~K}, 360 \mathrm{~K}$ and $370 \mathrm{~K})$ and three different concentrations of TMAO $(0 \mathrm{M}, 1 \mathrm{M}$ and $2 \mathrm{M})$ were chosen for a total of twelve different simulation conditions. For each of the simulations, a structure of PRB (PDB ID 1PRB) was mutated according to the fast-folding sequence shown below using the Mutator Plugin in VMD. ${ }^{32}$ The simulations were conducted in the NVT ensemble, with a $2.5 \mathrm{fs}$ time step, and a $1 \mathrm{~nm}$ cutoff for Lennard-Jones interactions. Electrostatic forces are calculated using the particle-mesh Ewald method. ${ }^{33}$ Temperature is held constant using a Nose-Hoover thermostat. ${ }^{34,35}$ Frames were saved every 100 ps and analyzed using the methods described below. The CHARMM36 force field ${ }^{36}$ was chosen to carry out the simulations. The protein was solvated with TIP3P water ${ }^{37}$ in a periodic box of size 57.36 $\times 57.36 \times 57.36 \AA^{3}$. NaCl $\left(2 \mathrm{Na}^{+}\right.$and $1 \mathrm{Cl}^{-}$ions) was used to balance out charges and standard protonation states were assumed for all the amino acid side chains.

Protein Sequence (PDB ID: 1PRB): LKNAIEDCIA ELKKAGITSD FVFNAWNKAK TVEEVNALVN EILKAHA

TMAO Force field. We started simulations by choosing three different force fields from Kast et al., ${ }^{38}$ Netz et al. ${ }^{18}$ and one generated by us from CGENff. ${ }^{39}$ The difference between Kast and CGENff was minimal, so we simulated only with the Kast and Netz force fields. The major difference between Kast and Netz is the distribution of partial charges, which could potentially change the interaction between the protein side chains and TMAO. From a simulation of $5 \mu$ s of PRB with $1 \mathrm{M} \mathrm{TMAO}$ with the two different force fields at $340 \mathrm{~K}$, we couldn't find any major differences so choose to use the more widely used force field (Kast) for rest of the simulations.

Analysis. Python $3.7^{40}$ and the molecular dynamics analysis package MDTraj ${ }^{41}$ were used for analysis. Volume calculations were performed in ProteinVolume. ${ }^{42}$ Solvent accessible surface area (SASA) calculations were done in $\mathrm{VMD}^{32}$ with a probe radius of $1.4 \AA$. They were normalized according to equation (1) with the SASA of the PDB structure. The pairwise distance measurements between two atoms for calculating radial distribution function were done by MDTraj, along with assignment of the nearest atom in contact. The volume occupied by shells around the protein was done by an in-house implementation of Monte Carlo method in VMD.

$$
S A S A_{\text {norm }}=\frac{S A S A(t)-S A S A_{P D B}}{S A S A_{P D B}}
$$

Fraction of Native contacts (Q). The determination of folded and unfolded ensembles was done by assigning a value $Q$ between 1 and 0 to each timeframe. Here 1 represents a completely folded structure, while 0 represents a completely unfolded structure. This value was calculated according to equation (2) as defined previously ${ }^{43,44}$ to indicate the number of native contacts preserved compared to a native state. The native state was determined by clustering the trajectory using RMSD by the gmx_cluster command in GROMACS ${ }^{31}$ separated by an RMSD difference of at least $4 \AA$. The largest cluster in the $340 \mathrm{~K} 0 \mathrm{M}$ TMAO trajectory was designated as the folded ensemble and native contacts maintained for at least eighty percent of the trajectory were used to calculate the $Q$ value for all the trajectories. Timeframes with a $Q$ value between 1 to 0.8 were defined as the folded structures, while values between 0.2 to 0 were defined as the unfolded structure to determine folded 
and unfolded ensembles. The value of $\alpha$ and $\beta$ was set to be 30 and 1.4 respectively to optimize for the difference between the folded and unfolded state.

$$
Q(t)=\frac{1}{N} \sum_{i, j} \frac{1}{1+\exp \left\{\alpha\left[d_{i j}(t)-\beta d_{i j}^{0}\right]\right\}},
$$

where $d_{i j}^{0}$ is the averaged native contact distance, $\alpha$ and $\beta$ are smoothing factors.

Hydrogen bond lifetimes and diffusion rates. The hydrogen bond lifetimes of water around the protein were calculated using analysis module in MDAnalysis ${ }^{45,46}$ package. The half-lives of the bonds were calculated by fitting the autocorrelation curves to a single exponential curve. The diffusion rate was calculated using the mean square displacement module and fitting the result to the equation (3).

$$
M S D=6 D t
$$

where $M S D$ is the mean square displacement, $D$ is the diffusion coefficient and $t$ is time.

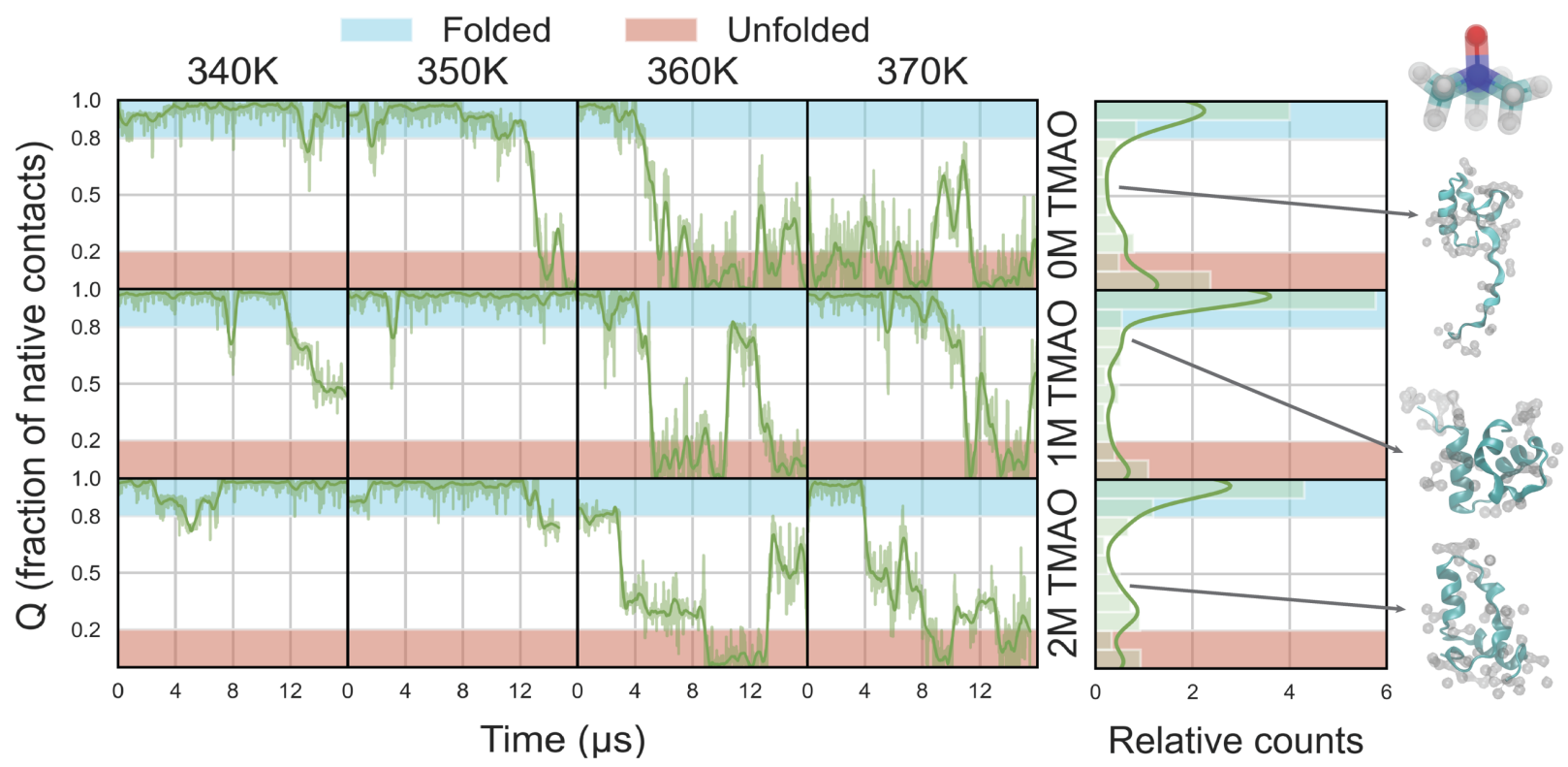

Fig. 1 Fraction of native contacts for all trajectories with different concentrations of TMAO $(0 \mathrm{M}, 1 \mathrm{M}$ and $2 \mathrm{M})$ and at different temperatures ( $340 \mathrm{~K}, 350 \mathrm{~K}, 360 \mathrm{~K}$ and $370 \mathrm{~K}$ ) are shown as moving average (solid green line) over 101 frames (101 ns) with a polynomial of degree 3 using a Savitzky-Golay filter. The light blue band indicates when the protein is in the folded state, while the light red band indicates protein in the unfolded state defined according to $Q$. The stick figure (top right) shows a TMAO molecule structure - oxygen (red), nitrogen (blue), carbon (light blue) and hydrogen (grey). The histograms (right) are binned together across all temperatures for a particular concentration of TMAO. Representative structure snapshots show the arrangement of water (grey) within $3 \AA$ around the protein (teal) at different $Q$ values.

\section{Results and Discussion}

A low concentration threshold of TMAO stabilizes protein against thermal denaturation. Fraction of native contacts $(Q)$ is a well-established measure of the foldedness of the protein. ${ }^{43}$ To this end we plot the timeseries for all the different temperatures and concentrations of TMAO used in our simulations (Fig. 1). The $Q$ values for folded structures are considered in the range 1 to 0.8 indicating close resemblance to the native state as defined by clustering of the $340 \mathrm{~K} 0 \mathrm{M}$ TMAO simulation. Similarly, the range of 0.8 to 0.2 is defined as the intermediate ensemble, while 0.2 to 0 
is the unfolded ensemble. On average, $Q$ decreases as the temperature increases in all simulations, most prominently in the $0 \mathrm{M}$ TMAO case. There are almost no folded structures present in the simulation at $370 \mathrm{~K}$ in $0 \mathrm{M}$ TMAO, highlighting that the protein is almost completely unfolded at this temperature. In the presence of TMAO, long-lived folded states are observed even at higher temperatures. However, the stabilization due to TMAO is not dependent on the concentration of TMAO higher than $1 \mathrm{M}$, with similar folded and unfolded time observed at $1 \mathrm{M}$ and $2 \mathrm{M} \mathrm{TMAO}$. This trend is consistent on other traditional reaction coordinates SASA, root mean square deviation (RMSD) and radius of gyration ( $\left.R_{\text {gyr }}\right)$ as can be seen in Fig. S1-3.

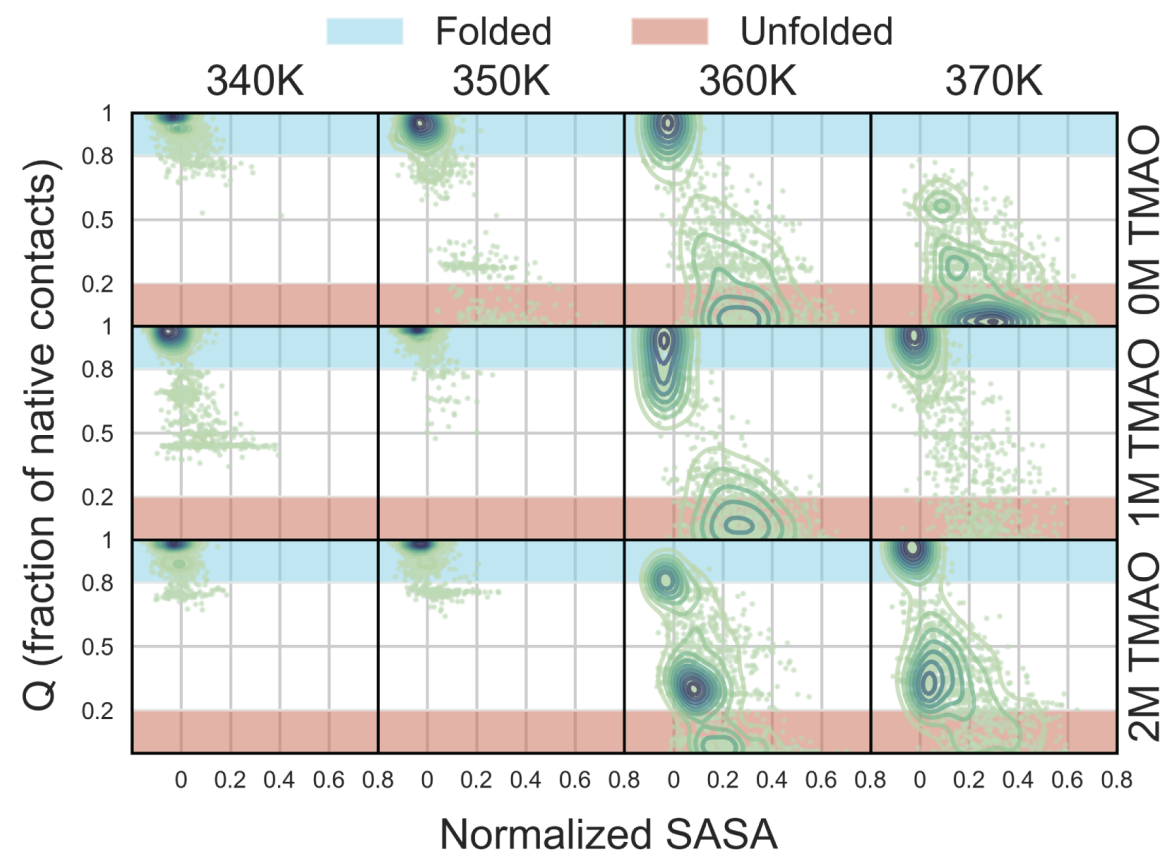

Fig. 2 Normalized solvent-accessible surface area (SASA) vs. fraction of native contacts $(Q)$ plotted as a scatter plot with kernel density estimator isocontours to indicate the number of distinct intermediates that protein $B$ might be going through between folded to unfolded state at different concentrations of TMAO and different temperatures. SASA is normalized compared to the SASA of the PDB structure by equation (1) used for the simulations. The light blue band indicates when the protein is in the folded state, while the light red band indicates protein in the unfolded state defined according to $Q$.

This is interesting because most osmolytes induce a concentration-dependent stabilization, ${ }^{4}$ but TMAO seems to saturate below $1 \mathrm{M}$. The maximum amount of TMAO that has been found in biological samples is actually below $1 \mathrm{M}^{7}$ Other studies have previously reported that TMAO can have a destabilizing effect at very high concentrations, ${ }^{47}$ so the concentration-independence between 1 and $2 \mathrm{M}$ may be a sign of the plateau between a threshold up to which the stabilizing effect increases, and a threshold where TMAO would destabilize the protein.

Increased foldedness in TMAO leads to lower solvent exposure. For all concentrations of TMAO, increasing unfoldedness leads to an increase in the amount of solvent accessible surface area (SASA), as is expected. However, there is a large spread for all values of $Q$ indicating rather large dynamical fluctuations of SASA. This is especially true for structures that have lower $Q$ values, indicating the presence of collapsed structures for unfolded proteins with SASA similar to that of the folded protein. The protein free energy surface appears to be rugged with several transient but longlived states that have been grouped together in our analysis as the intermediate state (Fig. 2). TMAO 
promotes population of multiple compact intermediate states over the unfolded state, which might help the protein refold more quickly and avoid aggregation when subjected to environmental stressors like temperature.

TMAO is thought to protect proteins from pressure by compacting the folded state by pushing water onto the protein surface. However, in our simulations the protein void volume does not change by a large amount (Fig. S4) in the presence of TMAO at a given temperature. We do not observe compaction of the folded state due to TMAO. There is also a shift in helical arrangement giving rise to high $Q$ values (0.6-0.8) in the presence of TMAO indicating a folded non-native state. This indicates a different mechanism of protein stabilization by TMAO with regards to thermal denaturation as compared to pressure denaturation. Even though thermal stabilization by TMAO is also associated with more water near the protein surface (Fig. S5), the mechanism of stabilization seems distinct between thermal and pressure denaturation, reflecting on pressure and volume being conjugate thermodynamic variables, while temperature and volume are not as closely linked.
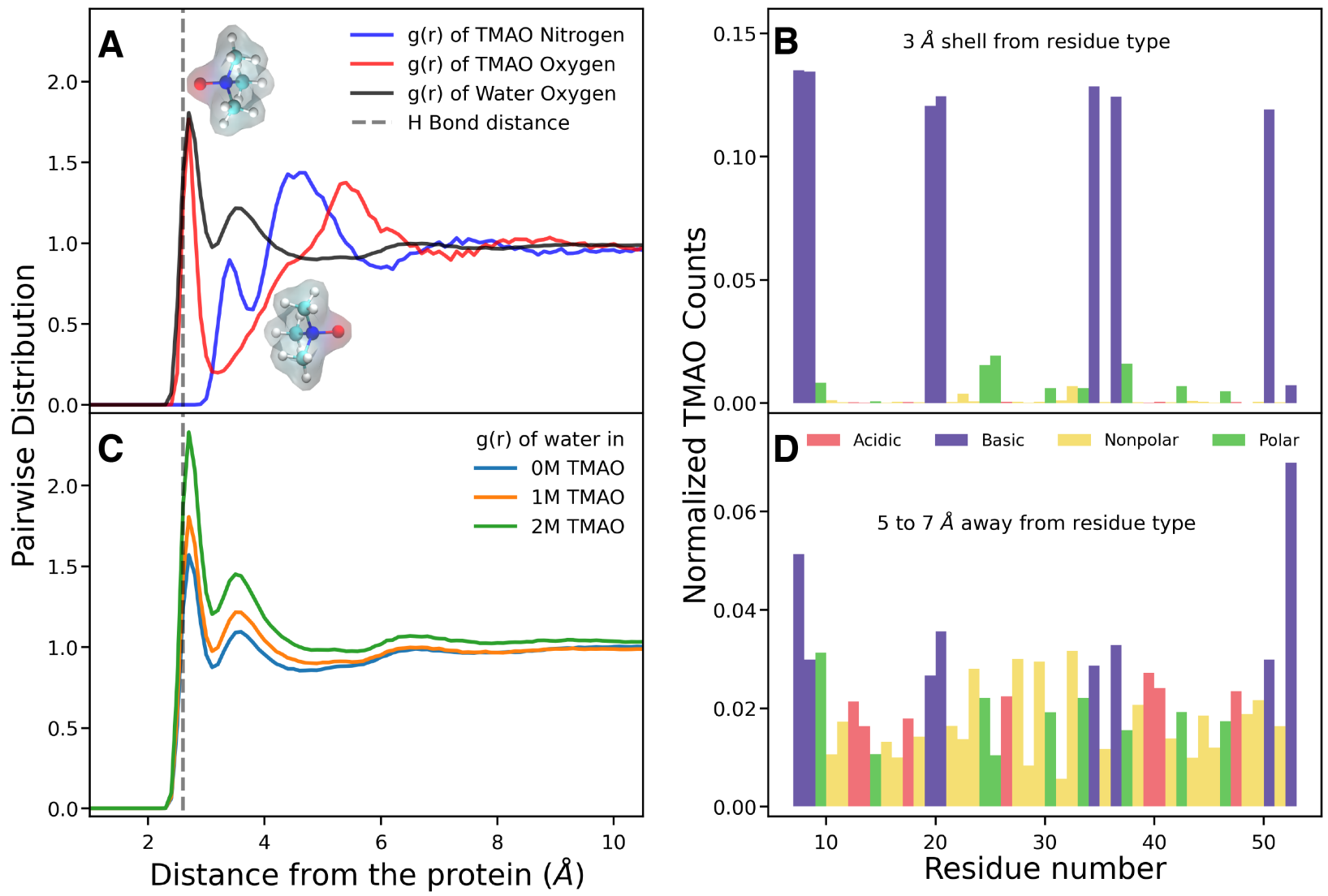

Fig. 3 Water and TMAO interactions with PRB for the simulation at $360 \mathrm{~K}$ and $1 \mathrm{M}$ TMAO. (A) The radial distribution function $g(r)$ is plotted versus distance from the protein surface (excluding hydrogens) to TMAO and water. To highlight the orientation of the TMAO towards the protein, two different distances are shown, from the nitrogen (blue) and the oxygen (red) atoms. The dashed line indicates a typical hydrogen bond distance from the protein surface. The images of TMAO molecules (inserts) are displaced and oriented towards the deduced mode of interaction that accounts for the visible peak. (B) The first TMAO peak at $2.7 \AA$ in $(A)$ shows a normalized contact count that favor basic residues strongly over all others. (C) Pairwise distance distribution of water from the PRB protein surface for the simulations at $360 \mathrm{~K}$ and $0 \mathrm{M}$ (light blue), $1 \mathrm{M}$ (orange) and $2 \mathrm{M}$ (green) TMAO. The trajectory has been divided into three distinct structural ensembles - folded (cyan), 
unfolded (dark red) and intermediate (grey). (D) The normalized count of residues nearest TMAO in the second peak at 5 to $6 \AA$ in $(A)$ shows that TMAO evenly distributed over the protein surface at that distance.

TMAO is mostly excluded from the protein surface but interacts with basic side chains. The radial distribution function of TMAO around the different ensembles of the protein at $360 \mathrm{~K}$ in $1 \mathrm{M}$ TMAO shows how TMAO organizes around the protein and influences the organization of water around the protein (Fig. 3).

The majority of TMAO molecules lies in a second solvation shell at 4 to $5 \AA$ (nitrogen) or 5 to $6 \AA$ (oxygen). The oxygen is more likely to point away from the protein (Fig. 3A), and the methyl groups are more likely to point towards the protein surface. To quantify whether this shell of TMAO molecules is evenly distributed over the protein surface or prefers certain side chains, we plotted a histogram of normalized count of TMAO nearest to each side chain (Fig. 3B and 3D). TMAO molecules in the second shell have no preferential interaction with any particular type of residue on the protein surface (Fig. 3D).

In contrast, the TMAO peak in the first solvation shell at 2.7 $\AA$ (Fig. 3A) reveals an additional more specific interaction of TMAO with the protein, even though most TMAO is excluded from the surface. To quantify this interaction, we again binned a normalized count of TMAO nearest to each side chain, this time for TMAO in the first shell (Fig. 3B). The partially charged oxygen atom of TMAO almost exclusively interacts with the basic side chains of the protein, with only minimal interaction with the other side chain types. Indeed the experimental transfer free energies of side chains in 1M TMAO from water are the lowest (most negative) with positively charged residues. ${ }^{48}$ In agreement with a spectroscopic study ${ }^{49}$ we observe that the methyl group of TMAO molecules in this first solvation shell tends to point away from the surface of the protein. TMAO is excluded from the protein surface mainly near hydrophobic side chains, in agreement with the exclusion measured by X-ray scattering ${ }^{50}$ and spectroscopy. ${ }^{51}$

The TMAO interactions with the three protein conformational ensembles (folded, intermediate, unfolded), (Fig. S5), indicate that the osmolyte does not interact preferentially with any of the protein ensembles. The increased amount of TMAO in the unfolded and intermediate ensemble compared with the folded ensemble in Fig. S5 can be accounted for simply by the increase in the solvent accessible surface area of unfolded and intermediate structures.

Water is pushed to the vicinity of protein $B$ in the presence of TMAO. The first shell of water in Fig. $3 A$ lies at $2.7 \AA$ and is due to direct interaction of water with the protein surface. These interactions are mostly hydrogen bonds between the side chains and backbone and water, along with other non-specific interactions.

The probability of water oxygen being present in the first solvation shell is increased in the presence of TMAO, critical for keeping the protein folded at higher temperature (Fig. 3C). Increased hydration may be promoted by the presence of the TMAO oxygen atoms in the vicinity of the protein surface. Additionally, TMAO appears to be hydrophilic in our simulations and actively interacts with waterboth the oxide moiety and the methyl group of TMAO are hydrated (Fig. 3A), which agrees with experimental density data ${ }^{52}$.

Thus, increased solvation by water at $2.7 \AA$ from the protein surface directly correlates to the cosolute effect of TMAO 'herding' water into the hydration shell to help keep protein B stable against denaturation. As can be seen, the amount of water pushed in is dependent on TMAO concentration 
- with $2 \mathrm{M}$ TMAO pushing in more water than 1M TMAO. The same saturation effect was discussed earlier in the context of protein stabilization by TMAO.

Water dynamics and hydrogen bond network around the protein. We selected five protein-water structures at $4 \mu \mathrm{s}$ intervals from simulations at $360 \mathrm{~K}$ for each TMAO concentration and performed fifteen short 100 ps MD simulations, with finer temporal sampling of the trajectory, to study the residence times and diffusion rates of water molecules around the protein in the presence and absence of TMAO. We found that the diffusion of water molecules does not significantly change in the presence of TMAO (Fig. 4A). A previous study has shown a decrease in the diffusion rate of both bulk water and water hydrogen bonded with TMAO, ${ }^{1}$ whereas we find that diffusion is more dependent on distance from the protein surface than on TMA concentration in Figure 4A. Intriguingly, the TMAO narrows the range of diffusion coefficients. TMAO evens out the diffusion of water molecules particularly in the main 5 to $6 \AA$ shell (not bonded to basic sidechains) where most TMAO resides. This is consistent with the 'herding' effect described above.
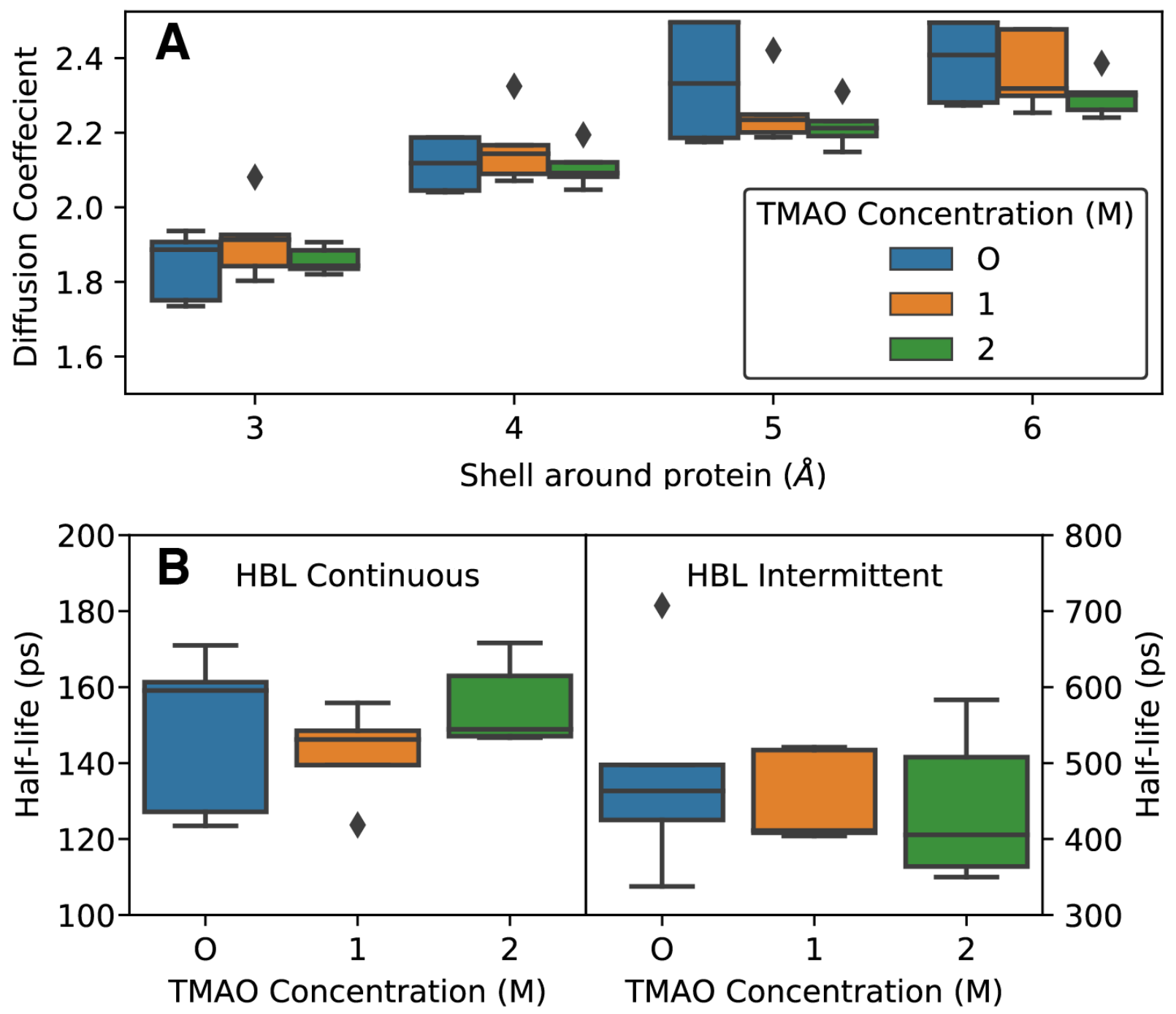

Fig. 4 Water dynamics and water-water hydrogen bonds. (A) The diffusion rate of water plotted as a boxplot for shells from the protein surface at $3,4,5$ and $6 \AA$ for the OM (blue), $1 \mathrm{M}$ (orange) and $2 \mathrm{M}$ (green) TMAO concentrations based on the fifteen 100 ps simulations at $360 \mathrm{~K}$. (B) The continuous and intermediate waterwater hydrogen bond half-life times plotted as a boxplot for the $3 \AA$ shell around the protein for the fifteen 100 
ps simulations at $360 \mathrm{~K}-5$ each with $0 \mathrm{M}$ (blue), $1 \mathrm{M}$ (orange) and $2 \mathrm{M}$ (green) TMAO. The box in boxplot shows the interquartile range, the whiskers show the minimum and maximum, the dashes within the box show the median while the diamonds are the outliers.

The water-water hydrogen bond lifetimes are also similar in the presence or absence of TMAO (Fig. 4B). We used two definitions of the lifetime to quantify the lifetimes: the 'Continuous' lifetimes report the half-life of a hydrogen bond that remains continuously formed while 'Intermittent' lifetimes allow for the bond to be broken briefly and formed again. While TMAO affects the water structure around the protein and increases solvation, it does not influence the speed of hydrogen bond breaking and making of water molecules around the protein, perhaps, due to its relatively small size and its hydrophilic nature. TMAO might be behaving as a hydrated hard sphere ${ }^{52}$ and a small size crowder $^{54,55}$. In analogy to Fig. 4A, TMAO again narrows the range of hydrogen bond half-lives, making the water behave more uniformly than expected for the bulk.

\section{Conclusion}

Molecular dynamics simulation shows that Protein B is stabilized in the presence of TMAO against temperature denaturation. The stabilization appears at rather low concentration (<1 M TMAO), with no further increase upon doubling the TMAO concentration. Thus, TMAO is efficient at pushing water to the protein surface, while water is depleted farther from the protein surface where TMAO resides. This 'herding' manifests itself in a narrowing of the $\mathrm{H}$-bond lifetime and diffusion coefficient distributions of water, rather than a change in average diffusion or lifetime. Studies in the presence of urea have suggested that TMAO can't form hydrogen bonds with protein. ${ }^{56}$ Urea and protein interaction would be stronger even in the presence of TMAO compared to protein TMAO interaction. Although most TMAO is excluded from the protein surface, there are interactions between the TMAO oxygen and basic side chains that produce a significant peak in the TMAO $g(r)$ near the protein surface. Due to the absence of urea in our system we see transient hydrogen bonds with the basic side chains of the protein. Unlike chaotropes, this binding is limited to side chains and not the backbone of the protein, and TMAO avoids hydrophobic patches on the protein surface. The interactions with basic residues might also play a role in the stabilization against pressure. One way to further probe this would be to study relative amino acid frequency in deep sea organisms. The mechanism of thermal stabilization is distinct but bears similarities to stabilization against pressure and urea in terms of exclusion from the surface of protein. At the level of overall protein structure, the stabilization is an increase in the population of compact intermediate states, making the low-lying protein free energy landscape rougher, ${ }^{52}$ while reducing the mostly unfolded population of protein. In that regard, TMAO acts like a small crowding agent.

\section{References}

(1) Yancey, P. H. Water Stress, Osmolytes and Proteins. Integr Comp Biol 2001, 41 (4), 699-709.

(2) Ball, P.; Hallsworth, J. E. Water Structure and Chaotropicity: Their Uses, Abuses and Biological Implications. Phys. Chem. Chem. Phys. 2015, 17 (13), 8297-8305.

(3) Sukenik, S.; Sapir, L.; Gilman-Politi, R.; Harries, D. Diversity in the Mechanisms of Cosolute Action on Biomolecular Processes. Faraday Discuss. 2013, 160, 225-237.

(4) Canchi, D. R.; García, A. E. Cosolvent Effects on Protein Stability. Annual Review of Physical Chemistry 2013, 64 (1), 273-293.

(5) Yancey, P.; Clark, M.; Hand, S.; Bowlus, R.; Somero, G. Living with Water Stress: Evolution of Osmolyte Systems. Science 1982, 217 (4566), 1214-1222. 
(6) Wang, A.; Bolen, D. W. A Naturally Occurring Protective System in Urea-Rich Cells: Mechanism of Osmolyte Protection of Proteins against Urea Denaturation ${ }^{\dagger}$. Biochemistry 1997, 36 (30), 9101-9108.

(7) Yancey, P. H.; Gerringer, M. E.; Drazen, J. C.; Rowden, A. A.; Jamieson, A. Marine Fish May Be Biochemically Constrained from Inhabiting the Deepest Ocean Depths. PNAS 2014, 111 (12), 4461-4465.

(8) Canchi, D. R.; Jayasimha, P.; Rau, D. C.; Makhatadze, G. I.; Garcia, A. E. Molecular Mechanism for the Preferential Exclusion of TMAO from Protein Surfaces. Journal of Physical Chemistry B 2012, 116 (40), 12095-12104.

(9) Julius, K.; Al-Ayoubi, S. R.; Paulus, M.; Tolan, M.; Winter, R. The Effects of Osmolytes and Crowding on the Pressure-Induced Dissociation and Inactivation of Dimeric LADH. Phys. Chem. Chem. Phys. 2018, 20 (10), 7093-7104.

(10) Lin, T.-Y.; Timasheff, S. N. Why Do Some Organisms Use a Urea-Methylamine Mixture as Osmolyte? Thermodynamic Compensation of Urea and Trimethylamine N-Oxide Interactions with Protein. Biochemistry 1994, 33 (42), 12695-12701.

(11) Zou, Q.; Bennion, B. J.; Daggett, V.; Murphy, K. P. The Molecular Mechanism of Stabilization of Proteins by TMAO and Its Ability to Counteract the Effects of Urea. J. Am. Chem. Soc. 2002, 124 (7), 1192-1202.

(12) Mondal, J.; Stirnemann, G.; Berne, B. J. When Does Trimethylamine N -Oxide Fold a Polymer Chain and Urea Unfold It? J. Phys. Chem. B 2013, 117 (29), 8723-8732.

(13) Smolin, N.; Voloshin, V.; Anikeenko, A.; Geiger, A.; Winter, R.; Medvedev, N. N. TMAO and Urea in the Hydration Shell of the Protein SNase. Phys. Chem. Chem. Phys. 2017.

(14) Ganguly, P.; Boserman, P.; Van Der Vegt, N. F. A.; Shea, J. E. Trimethylamine NOxide Counteracts Urea Denaturation by Inhibiting Protein-Urea Preferential Interaction. Journal of the American Chemical Society 2018, 140 (1), 483-492.

(15) Ganguly, P.; Polak, J.; van der Vegt, N. F. A.; Heyda, J.; Shea, J.-E. Protein Stability in TMAO and Mixed Urea-TMAO Solutions. J. Phys. Chem. B 2020, acs.jpcb.0c04357.

(16) Su, Z.; Dias, C. L. Individual and Combined Effects of Urea and Trimethylamine NOxide (TMAO) on Protein Structures. Journal of Molecular Liquids 2019, 293, 111443.

(17) Hilaire, M. R.; Abaskharon, R. M.; Gai, F. Biomolecular Crowding Arising from Small Molecules, Molecular Constraints, Surface Packing, and Nano-Confinement. J. Phys. Chem. Lett. 2015, 6 (13), 2546-2553.

(18) Schneck, E.; Horinek, D.; Netz, R. R. Insight into the Molecular Mechanisms of Protein Stabilizing Osmolytes from Global Force-Field Variations. J. Phys. Chem. B 2013, 117 (28), 8310-8321.

(19) Munroe, K. L.; Magers, D. H.; Hammer, N. I. Raman Spectroscopic Signatures of Noncovalent Interactions Between Trimethylamine N-Oxide (TMAO) and Water. J. Phys. Chem. B 2011, 115 (23), 7699-7707.

(20) Larini, L.; Shea, J. E. Double Resolution Model for Studying TMAO/Water Effective Interactions. Journal of Physical Chemistry B 2013, 117 (42), 13268-13277.

(21) Zetterholm, S. G.; Verville, G. A.; Boutwell, L.; Boland, C.; Prather, J. C.; Bethea, J.; Cauley, J.; Warren, K. E.; Smith, S. A.; Magers, D. H.; et al. Noncovalent Interactions between Trimethylamine $N$-Oxide (TMAO), Urea, and Water. J. Phys. Chem. $B$ 2018, 122 (38), 8805-8811. 
(22) Boob, M.; Wang, Y.; Gruebele, M. Proteins: "Boil 'Em, Mash 'Em, Stick 'Em in a Stew". J. Phys. Chem. B 2019, 123 (40), 8341-8350.

(23) Privalov, P. L.; Khechinashvili, N. N. A Thermodynamic Approach to the Problem of Stabilization of Globular Protein Structure: A Calorimetric Study. Journal of Molecular Biology 1974, 86 (3), 665-684.

(24) Lins, L.; Brasseur, R. The Hydrophobic Effect in Protein Folding. FASEB j. 1995, 9 (7), 535-540.

(25) Liu, F.; Nakaema, M.; Gruebele, M. The Transition State Transit Time of WW Domain Folding Is Controlled by Energy Landscape Roughness. J. Chem. Phys. 2009, 131 (19), 195101.

(26) Johansson, M. U.; de Château, M.; Wikström, M.; Forsén, S.; Drakenberg, T.; Björck, L. Solution Structure of the Albumin-Binding GA Module: A Versatile Bacterial Protein Domain. Journal of Molecular Biology 1997, 266 (5), 859-865.

(27) Lindorff-Larsen, K.; Piana, S.; Dror, R. O.; Shaw, D. E. How Fast-Folding Proteins Fold. Science 2011, 334 (6055), 517-520.

(28) Voloshin, V.; Smolin, N.; Geiger, A.; Winter, R.; Medvedev, N. N. Dynamics of TMAO and Urea in the Hydration Shell of the Protein SNase. Phys. Chem. Chem. Phys. 2019, 21 (35), 19469-19479.

(29) Mondal, J.; Stirnemann, G.; Berne, B. J. When Does TMAO Fold a Polymer Chain and Urea Unfold It? 2013.

(30) Guin, D.; Mittal, S.; Bozymski, B.; Shukla, D.; Gruebele, M. Dodine as a KosmoChaotropic Agent. J. Phys. Chem. Lett. 2019, 10 (10), 2600-2605.

(31) Abraham, M. J.; Murtola, T.; Schulz, R.; Páll, S.; Smith, J. C.; Hess, B.; Lindahl, E. GROMACS: High Performance Molecular Simulations through Multi-Level Parallelism from Laptops to Supercomputers. SoftwareX 2015, 1-2, 19-25.

(32) Humphrey, W.; Dalke, A.; Schulten, K. VMD: Visual Molecular Dynamics. Journal of Molecular Graphics 1996, 14 (1), 33-38.

(33) Essmann, U.; Perera, L.; Berkowitz, M. L.; Darden, T.; Lee, H.; Pedersen, L. G. A Smooth Particle Mesh Ewald Method. J. Chem. Phys. 1995, 103 (19), 8577-8593.

(34) Nosé, S. A Molecular Dynamics Method for Simulations in the Canonical Ensemble. Molecular Physics 1984, 52 (2), 255-268.

(35) Hoover, W. G. Canonical Dynamics: Equilibrium Phase-Space Distributions. Phys. Rev. A 1985, 31 (3), 1695-1697.

(36) Huang, J.; MacKerell, A. D. CHARMM36 All-Atom Additive Protein Force Field: Validation Based on Comparison to NMR Data. J Comput Chem 2013, 34 (25), 2135-2145.

(37) Mark, P.; Nilsson, L. Structure and Dynamics of the TIP3P, SPC, and SPC/E Water Models at 298 K. J. Phys. Chem. A 2001, 105 (43), 9954-9960.

(38) Kast, K. M.; Brickmann, J.; Kast, S. M.; Berry, R. S. Binary Phases of Aliphatic NOxides and Water: Force Field Development and Molecular Dynamics Simulation. J. Phys. Chem. A 2003, 107 (27), 5342-5351.

(39) Vanommeslaeghe, K.; Hatcher, E.; Acharya, C.; Kundu, S.; Zhong, S.; Shim, J.; Darian, E.; Guvench, O.; Lopes, P.; Vorobyov, I.; et al. CHARMM General Force Field (CGenFF): A Force Field for Drug-like Molecules Compatible with the CHARMM All-Atom Additive Biological Force Fields. J Comput Chem 2010, 31 (4), 671-690.

(40) Anaconda Software Distribution; Anaconda, Inc, 2020. 
(41) McGibbon, R. T.; Beauchamp, K. A.; Harrigan, M. P.; Klein, C.; Swails, J. M.; Hernández, C. X.; Schwantes, C. R.; Wang, L.-P.; Lane, T. J.; Pande, V. S. MDTraj: A Modern Open Library for the Analysis of Molecular Dynamics Trajectories. Biophysical Journal 2015, 109 (8), 1528-1532.

(42) Chen, C. R.; Makhatadze, G. I. ProteinVolume: Calculating Molecular van Der Waals and Void Volumes in Proteins. BMC Bioinformatics 2015, 16 (1), 101.

(43) Best, R. B.; Hummer, G.; Eaton, W. A. Native Contacts Determine Protein Folding Mechanisms in Atomistic Simulations. Proceedings of the National Academy of Sciences 2013, 110 (44), 17874-17879.

(44) Sukenik, S.; Pogorelov, T. V.; Gruebele, M. Can Local Probes Go Global? A Joint Experiment-Simulation Analysis of $\lambda$ 6-85 Folding. The Journal of Physical Chemistry Letters 2016, 7 (11), 1960-1965.

(45) Michaud-Agrawal, N.; Denning, E. J.; Woolf, T. B.; Beckstein, O. MDAnalysis: A Toolkit for the Analysis of Molecular Dynamics Simulations. J. Comput. Chem. 2011, 32 (10), 2319-2327.

(46) Gowers, R.; Linke, M.; Barnoud, J.; Reddy, T.; Melo, M.; Seyler, S.; Domański, J.; Dotson, D.; Buchoux, S.; Kenney, I.; et al. MDAnalysis: A Python Package for the Rapid Analysis of Molecular Dynamics Simulations; Austin, Texas, 2016; pp 98-105.

(47) Rani, A.; Jayaraj, A.; Jayaram, B.; Pannuru, V. Trimethylamine- N -Oxide Switches from Stabilizing Nature: A Mechanistic Outlook through Experimental Techniques and Molecular Dynamics Simulation. Sci Rep 2016, 6 (1), 1-14.

(48) Auton, M.; Bolen, D. W. Predicting the Energetics of Osmolyte-Induced Protein Folding/Unfolding. PNAS 2005, 102 (42), 15065-15068.

(49) Sagle, L. B.; Cimatu, K.; Litosh, V. A.; Liu, Y.; Flores, S. C.; Chen, X.; Yu, B.; Cremer, P. S. Methyl Groups of Trimethylamine N-Oxide Orient Away from Hydrophobic Interfaces. J. Am. Chem. Soc. 2011, 133 (46), 18707-18712.

(50) Stanley, C.; Rau, D. C. Assessing the Interaction of Urea and Protein-Stabilizing Osmolytes with the Nonpolar Surface of Hydroxypropylcellulose ${ }^{\dagger}$. Biochemistry 2008, 47 (25), 6711-6718.

(51) Sagle, L. B.; Cimatu, K.; Litosh, V. A.; Liu, Y.; Flores, S. C.; Chen, X.; Yu, B.; Cremer, P. S. Methyl Groups of Trimethylamine N-Oxide Orient Away from Hydrophobic Interfaces. J. Am. Chem. Soc. 2011, 133 (46), 18707-18712.

(52) Rösgen, J.; Jackson-Atogi, R. Volume Exclusion and H-Bonding Dominate the Thermodynamics and Solvation of Trimethylamine- $\mathrm{N}$-Oxide in Aqueous Urea. $\mathrm{J}$. Am. Chem. Soc. 2012, 134 (7), 3590-3597.

(53) Teng, X.; Huang, Q.; Dharmawardhana, C. C.; Ichiye, T. Diffusion of Aqueous Solutions of Ionic, Zwitterionic, and Polar Solutes. The Journal of Chemical Physics 2018, 148 (22), 222827.

(54) Hilaire, M. R.; Abaskharon, R. M.; Gai, F. Biomolecular Crowding Arising from Small Molecules, Molecular Constraints, Surface Packing, and Nano-Confinement. J. Phys. Chem. Lett. 2015, 6 (13), 2546-2553.

(55) Davis, C. M.; Gruebele, M.; Sukenik, S. How Does Solvation in the Cell Affect Protein Folding and Binding? Current Opinion in Structural Biology 2018, 48, $23-29$.

(56) Yang, Y.; Mu, Y.; Li, W. Microscopic Significance of Hydrophobic Residues in the Protein-Stabilizing Effect of Trimethylamine N-Oxide (TMAO). Phys. Chem. Chem. Phys. 2016, 18 (32), 22081-22088. 